\title{
A Novel Approach for Farming using loT
}

\author{
Bhushan Jadhav, PhD \\ Thadomal Shahani \\ Engineering College \\ Mumbai 400050
}

\author{
Rishabh Kriplani \\ Thadomal Shahani \\ Engineering College \\ Mumbai 400050
}

\author{
Rahul Badugu \\ Thadomal Shahani \\ Engineering College \\ Mumbai 400050
}

\author{
Ritesh Keswani \\ Thadomal Shahani \\ Engineering College \\ Mumbai 400050
}

\begin{abstract}
Farming is India's most critical and basic profession, combining all food needs with importance to humanity and the supply of essential raw materials for many businesses. But in this digitized world, there are less opportunities available with the farming when it considers manual work. The emerging technologies like IoT, Cloud Computing and Big data analytics has made a significant improvement in the current IT applications. However, there is a huge scope of introducing these technologies into current farming practices. These technologies are highly compatible to be leverage in current farming process. The automation in farming with these technologies can reduce both manpower and cost. The growth of revolutionary farming techniques using emerging technologies is gradually improving crop production, making it more productive and reducing irrigation waste. So, in this research paper, a modern farming system is introduced with proposed solutions that can overcome the existing challenges and limitations along with assistance in proper water management, crop management and provide ideal crop and fertiliser recommendations based on historic soil condition results.
\end{abstract}

\section{Keywords}

IoT, Soil moisture sensor, Node MCU, DHT-11, Machine learning.

\section{INTRODUCTION}

Agriculture can be a significant part of India's GDP, with an 18 percent share and $50 \%$ of the country's population are working. As farming grows exponentially, 45 farmers commit suicide in the country every day thanks to numerous main issues. The tendency of suicidal farmers in our country has risen over the past nearly 20 years. 11.2 percent of all suicides in the country were reflected by farmer suicides according to a National Crimes Report Bureau report [1]. It's a serious issue. Researchers have isolated certain trends and explanations in recent studies for clarifying this number. Indian farmers 76 percent. The study of the Food and Agriculture Association, the global organization survey. Production for the population that is evolving should be increased by $70 \%$ by 2050 . The foundation of humanity is agriculture since it is the first source of food and food in the growth of national economies, it plays an important role. Once in a while, farmers had great control of crops as a result of unexpected and demanding environmental conditions. The research in this report was strongly inspired by the awareness of the potential water crisis which would cause a global catastrophe. Farming in India is a huge occupation and requires huge volumes of water in these areas. Water services are used only for irrigation more than 80 percent. This current pattern will have an absolutely declining impact on water availability. In view of this, a design to regulate the use of water has been suggested. Incorporating smart technologies in farming practices would concentrate on improving land quality [3]. The traditional irrigation system is run by hand and is dependent on manually observed conditions in real time and on ground conditions.

The Current limitations of manual farming are given as follows:

Poor water management: Farmers face a major challenge with a shortage of water due to lower rainfall. Because of the difference in availability, farmers often have to water the field in strange periods. Over-watering and under-watering was often due to human error in few cases.

Poor Crop management and Low Fertility of Soils: Soil fertility must be improved, but the use of chemical fertilizers is restricted due to a lack of sufficient soil moisture. [8].

No crop recommendation features available based on historic soil condition results: Currently no data is available or is being collected to suggest a crop based on historic soil condition results.

The decrease in farm workforce has prompted the adoption of solutions for internet connectivity in agricultural practices to reduce manual labor. IoT solutions aim to help farmers to close the demand gap through high returns, profitability and environmental protection. The IoT technology approach is called precision agriculture in order to ensure optimum use of resources in order to achieve high crop production and to reduce operational costs. Specialized equipment, wireless connectivity, software and IT services are included in IoT in agricultural technologies. Smart farming based on IoT enables farmers and growers, from the quantity of fertilizer used to the number of journeys carried out by agricultural vehicles, to reduce waste, and improve the productivity of resources including water, electricity, and more. Smart farming based on IoT solutions is a system which has been developed for monitoring and automating irrigation systems in crop fields with the help of sensors (light, humidity, heat, soil moisture, crop health, etc.). Farmers are able to monitor the field from anywhere [7]. For instance, the farmer may deploy sensors to initiate the irrigation if the soil humidity level decreases. Compared to the conventional approach, smart farming is extremely efficient. The Fig. 1 shows the Scope of IoT in agriculture for recent and future.

In this research paper, a system is proposed which can overcome above limitations and will provide additional benefits to the current farming systems. This research paper has proposed a decision tree algorithm for calculating the percent of soil moisture with the help of humidity in percent and temperature in degree celsius. 


\section{INTERNET OF THINCS (IOT) IN ACRICULTURE MARKE} BY SYSTEM

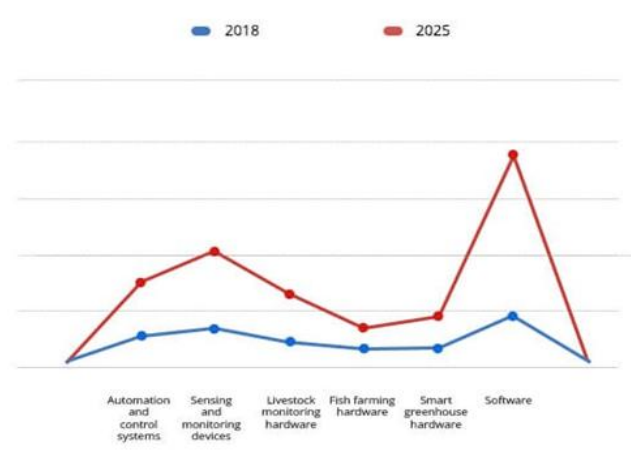

Fig 1: Scope of IoT in agriculture for recent and future

\section{RELATED WORKS}

Increasing production in the sector is a critical means of increasing farm income and satisfying the growing demand for food by rapidly increasing worldwide. A farm's productivity can be increased by deciding which crop variety produced the most productive production under complex soil, conditions of environment and irrigation. The most recent scenario of water tables declining, river bed extraction and volatile environment worsens every day.[1]

Rao R., and Sridhar B [4], takes advantage of the sensor of LM35, which is used for the tracking of water supplementations and is deployed throughout the sector. Suggested an LM35 temperature sensor, humidity sensor, RPi 3 model B, IC 3208 converter and relay and buzzer [9]. According to the above listed proposal, a $2.4 \mathrm{v}$ threshold value is ready for moisture in the soil; it might differ between crops. If this value is not detected, the soil is measured as dry $(2.4 \mathrm{v}$ in this case), and a signal is transmitted to the water pump. The water pump would be turned on. If not, the soil is measured as moist and the engine is turned OFF. The knowledge gained from sensors is absorbed into the cloud and can be reached from a handheld PC for farmers. The aim is to minimize water loss, function and increase efficiency in the survey presented in [5]. Moisture sensor is used to detect the moisture content in the soil and transmit information from the humidity sensor to Arduino. Moisture sensor is used for moisture sensing in the field. It operates on the free and short circuit theory. The circuit acts as an outer circuit when the surface is dry, shutting when the earth is warm. A Wi-Fi module for transmitting data from a sensor layer into the cloud is used for connectivity. Data from moisture sensors is sent to Arduino and the data or values are uploaded to the cloud via Wi-Fi by Arduino. The threshold value shall be calculated as required by the crop. The level of humidity tested with regard to the threshold value specified. For different crops, the threshold value is different. If the value of moisture is less than that of the reference value compressor, it stays OFF otherwise. This leads to water consumption reduction.

The key pitfalls of previous literature work were: 1) The data and crop conditions obtained were based primarily on sensors that were not reliable, and were susceptible to injury. 2) These networks of wireless sensors primarily rely on the Internet which could cause a significant disadvantage in remote locations; 3) Automatic irrigation systems have been expensive and in significant cases failed, since only a few threshold values have been historically enthusiastic about. 4) Smart farming technologies are available, but they can only use a limited number of IoT-specific instruments and will not be able to help data analyses and sharing. To interpret this data generated by human interaction requires a great amount of work to manually combine and compare the data collected from different sensors. These serious disadvantages included the inclusion of convolutional neural networks to efficiently investigate results. The paper suggests a novel IoT + ML approach, in which the MQTT protocol is used to connect devices in remote sites; weather data obtained is not sensor related; crop and mineral suggested consideration of present and past field data; crop condition is evaluated using real-time image recognition techniques; automatic irrigation is completed [1].

S. Geetha, P. Deepalakshmi, Shilpa Pande, [2] proposed a new idea of Smart Crop Monitoring System implemented using Internet of Things for sensing environmental conditions and forwarding the data, Machine Learning to generate decisions for crop management based on the data, Cloud for storage and an Android application interface for operation of the system. In order to schedule and digitize their farming practices, Smart Agro can help farmers. The farmers can therefore track crop requirements and forecast their growth accurately. This idea would certainly speed up their activities and make them more successful.

Aashu Bedrae R. K1, Jayalakshmi A2, Nayana M3, Swetha D4, Shridhara Y5 in [6] proposed a smart irrigation system which is soil sensor-based and run on the basis of soil moisture content. The soil humidity sensor is attached to Arduino's microcontroller input pins in this device. Sensor values are seen on the Smartphone. The pump is automatically turned ON/Off by the motor driver when the sensed value is greater than the software threshold values. The farmer is told by the smart telephone application and posted on the Web page about the current conditions in the area. By using this device, the farmer will at any time have access to information on the state of the field.

\section{PROPOSED SOLUTION}

The proposed solution consists of different modules which help to overcome the current issues in farming. In this approach to farming system, the data gathered from smart agricultural sensors are the key components, including sensors, control systems, automated hardware, variable rates technology, motion detectors, button camera and wearable instruments. These data are used to monitor the status of the farming as well as performance and efficiency of the equipment. The ability to predict production output enables the plan to better distribute the products. Smart farming predictive analytics, Predicting crops plays a crucial role, helping farmers decide on future plans for cultivation, storage, marketing and risk management [7]. This solution uses the different modules of IOT sensors and controllers to automate the farming process. In this paper, the solution is designed which uses few sensors which gives the amount of moisture within the soil, the humidity and temperature of the region. All these sensors along with NodeMCU are connected to the internet and a smartphone and real-time data is fetched from them.[10] Temperature, soil moisture sensor and NodeMCU are the key components of the proposed system architecture. NodeMCU has a significant role to play in the system. The DHT11 and Soil moisture sensors are deployed in the field 
and are connected to NodeMCU. The sensed data are sent to NodeMCU by these sensors. The algorithm for the decision tree is used in the data collection to estimate the exact results. All data sent from the sensors to the NodeMCU are stored in a database for future use. The block diagram of proposed system is shown in Fig.2.

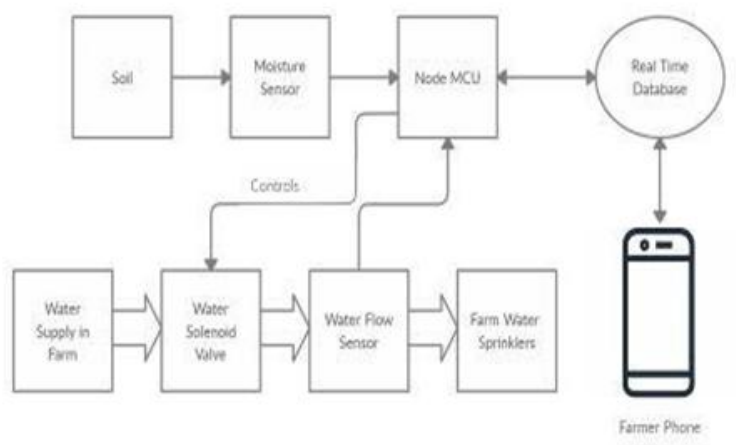

Fig 2: Block diagram of proposed system

The proposed solution requires following hardware and software components:

\subsection{Hardware Components}

a. NodeMCU: The NodeMCU is basically an open-source firmware, and also commonly used term for the popular ESP8266 Development Board, which is a low cost, development board designed for IoT applications.

\section{b. Sensors}

i. DHT-11: It is a simple digital temperature and humidity sensor at ultra-low cost. It uses a capacitive humidity sensor and a thermistor to measure the surrounding air, and spits out a digital signal on the data pin (no analog input pins needed). It's very easy to use, but takes careful time to collect data.

This sensor is used to read the temperature and humidity near the crop. Fig. 3 shows DHT-11 sensor.

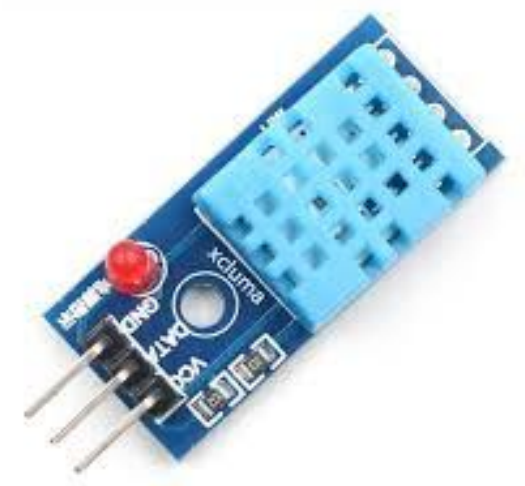

Fig 3: DHT11 sensor

ii. Soil Moisture Sensor: The moisture content in soil in which the plant is maintained is detected via this sensor. It has two electrodes which are placed in the soil. Simply insert this rugged sensor into the soil to be tested, and the volumetric water content of the soil is reported in percent. Fig. 4 shows soil moisture sensor.

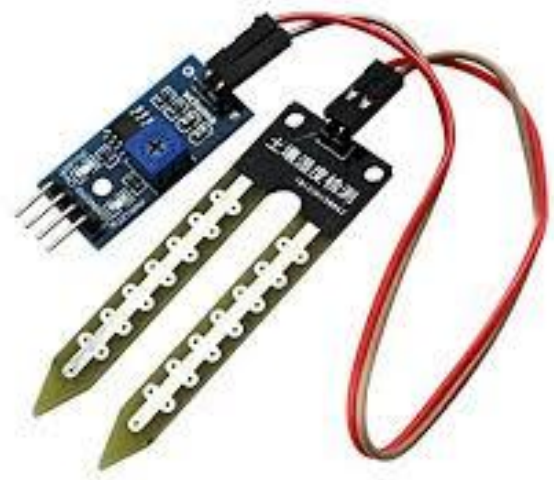

Fig 4: Soil Moisture Sensor

c. 5V Relay Module: High voltage handling capacity of 220v ac with full protection. It has a red LED that light up when a signal on the IN pin is received.

Once a signal is received, the relay triggers the output pins with an audible click. Fig. 5 shows relay module.

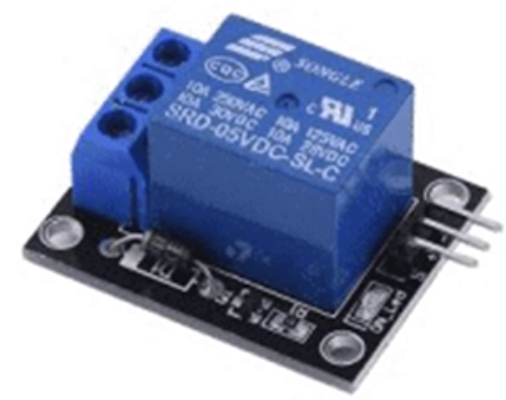

Fig 5: Relay Module

\subsection{Software Components}

a. Arduino IDE: A cross-platform (windows, MacOS, Linux) application, written for functionality in $\mathrm{C}$ and $\mathrm{C}++$, is the Arduino Integrated Development Environment (IDE). It is used to write and upload programs to compatible Arduino boards, but also to other vendor development boards with the help of core third parties.

b. Android Studio: The Android Studio, which was developed using JetBrains' IntelliJ IDEA software and specifically developed to Android development, is the official integrated development environment for Google's Android operation system. It helps you build, improve, and grow your app.

c. FireBase: Firebase is a Google platform for mobile and web application building.

The proposed system collects the data from different sensors and store it for analysis. The machine learning algorithm used in proposed system is a supervised deep learning algorithms, through which the machine already learns the responses, to learn the patterns involved. It analyses the various types of data, responds to each query, and involves a pattern. This process is known as data training. The bigger the details, the more detailed the findings are. The following stage includes the checking of the results. At this point, the machine to fix 
problems is given the most appropriate solution since the machine has knowledge of the problem-solving pattern and potential solutions. The accuracy of the result is calculated as previously stated by the data size, the algorithms used in the data and other considerations, such as noise and the outsourcing in the data provided as training information. The two main stages for every grouping are understanding and estimation. The model is generated in the learning process by means of feed training info. The model should predict the results in the prediction process based on the training data. A powerful classification algorithm can be used to perceive and analyze the decision tree algorithm. [3]

One of the effective and basic algorithms for smart agriculture is within the supervised learning family of algorithms is the decision tree algorithm. Unlike other algorithms in supervised learning, the decision tree algorithm is used to address multiple regression and classification problems. The main aim is to train a model that can predict the value or class of the goal variable by making simple and straightforward decisions based on previous data, i.e., training data. To predict a register class name, the first node that is the root node of the decision tree is required to begin. The attribute of the record should be checked with each root attribute value. Based on the validations, a path containing branches are followed with the matching value and jump to the succeeding node. In the proposed solution, an Android app is developed that allows us to display and remotely overview the soil sensor data. The app receives the data through the Wi-Fi module which is inbuilt in NodeMCU. The application was designed using Android Studio and Firebase. The application gives us real-time info, which will let us know the state of irrigation in the fields.

Delta: The total quantity of water required for any crop during its base period for its full-fledged nourishment when expressed in depth of water (i.e. in 'cm' or in 'inches') is called its Delta. Every crop has different growth requirement. Some quantity of water is required for any crop to come to its maturity. The Delta of the crop differs for different types of crops. The formulas for various calculations are given below.

Total amount of water required for a $\operatorname{crop}(\mathrm{T})=($ Delta $*$ Area of crop)/1000 liters

No. of days required for the growth of the crop $=\mathrm{n}$ days' Time interval for irrigation $=\mathrm{i}$ days

Total no. of irrigation $(\mathrm{Ni})=\mathrm{n} / \mathrm{i}$ times

Amount of water required per interval $=\mathrm{T} / \mathrm{Ni}$ liters

The moisture content of the soil is measured using a moisture sensor. It is fed to the NodeMCU which checks that the moisture content is less than the threshold or not. If it is less than the threshold, it signals the solenoid valve to open the valve and the relay module activates the submersible water pump and the water starts flowing. Now the flowing water will be measured by a waterflow sensor and a calculated amount of water will be supplied to the farm.

The prototype of proposed system runs in real time with a soil sensor. The sensor of the ground senses moisture in the ground. Based on soil and plant type, a threshold is preset in the template. As humidity falls below the set level, turn the machine on and supply the crops with water as soon as the ground humidity crosses above the threshold, the water supply ceases. Therefore, no human interference is required for the whole device automatically. Based on the moisture in the ground, the machine automatically supplies water. An application on our smartphone provides an explanation of the whole operation.

The possible advantages of proposed system are given as follows. [7]

a) Affordable- Open-source technology is used and much lower maintenance is needed. It can be incorporated without extra costs into the existing irrigation system.

b) Efficiency reduction: The system can operate $5 \mathrm{~V}$ Low power consumption (excluding pump). The power supply should also be tied to a residence or battery.

c) Help in water management -A balanced supply of water; no over water supply or drinking supply is available.

d) No human interference -The Smart farming system is fully automated, and thus requires no workforce.

e) Remote Control - The system can be operated remotely using the established app via smartphones and data can be accessed from anywhere over the Internet.

\section{RESULTS AND ANALYSIS}

In the proposed system, the different modules of IOT sensors and controllers are used to automate the farming process. The different sensors collect the data which is required to perform the analysis for getting the insights and desired outcomes. The proposed system has DHT11 sensor to collect the temperature of the soil. The humidity of the soil is also being computed with the help of sensors. Based on the temperature and the humidity the soil moisture is derived by the sensor.

The Table 1: shows the reading of temperature and humidity along with derived soil moisture in percentage.

Table 1: Reading of temperature and humidity along with derived soil moisture in percentage

\begin{tabular}{|c|c|c|c|}
\hline $\begin{array}{c}\text { Sr. } \\
\text { No. }\end{array}$ & $\begin{array}{c}\text { Humidity } \\
(\mathbf{\%})\end{array}$ & $\begin{array}{c}\text { Temperature } \\
\left({ }^{\circ} \mathbf{C}\right)\end{array}$ & Soil Moisture (\%) \\
\hline 1. & 61 & 39 & 47 \\
\hline 2. & 68 & 48 & 75 \\
\hline 3. & 73 & 30 & 63 \\
\hline 4. & 76 & 29 & 67 \\
\hline 5. & 75 & 36 & 81 \\
\hline 6. & 92 & 51 & 37 \\
\hline 7. & 74 & 44 & 65 \\
\hline 8. & 90 & 49 & 35 \\
\hline 9. & 62 & 47 & 74 \\
\hline 10. & 67 & 53 & 46 \\
\hline
\end{tabular}

The correlation between humidity, temperature and soil moisture is shown in Fig. 6. 


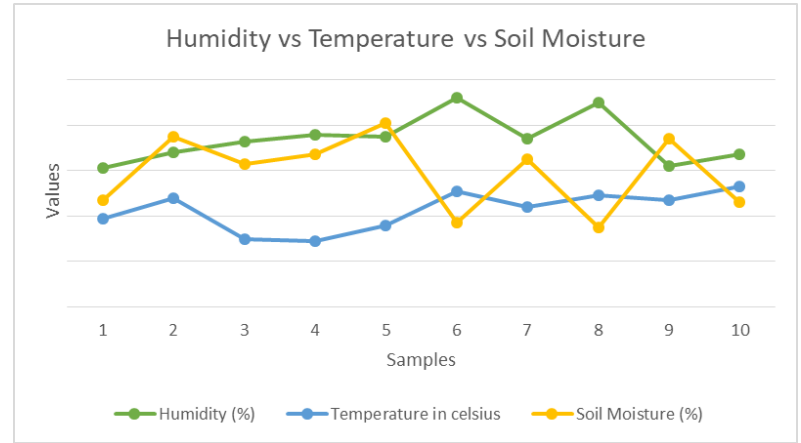

Fig 6: Correlation between humidity, temperature and soil moisture

From Fig.6 it can be observed that, as soon as the humidity level rises the soil moisture increases while rise in the temperature level may evaporate the moisture in soil but because of high humidity the moisture in the soil retained to its higher level.

The proposed solution is intended to give maximum benefits to farmers in terms of providing automation through an android application. It reduces the human interference and manpower at reduced costs.

The proposed solution has Android Application to interact with farm sensors and show real time results to the farmers. Fig. 7 shows the temperature, humidity and moisture content fetched in real time.

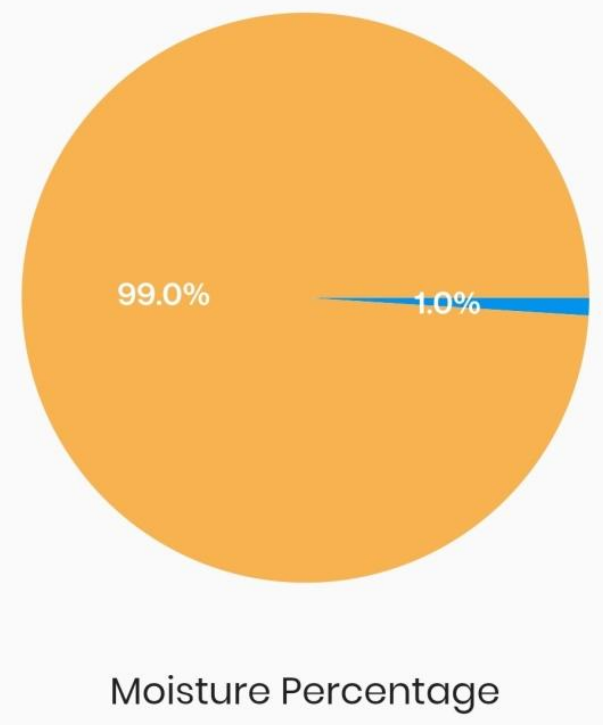

\section{Humidity: 62 Temp: 25.4}

$$
\text { fetch }
$$

Fig 7: Temperature, humidity and moisture content fetched in real time

The proposed system also suggests the crop based on the location, humidity with temperature and soil moisture value. The Fig. 8 shows the crop prediction based on location and soil characteristics.

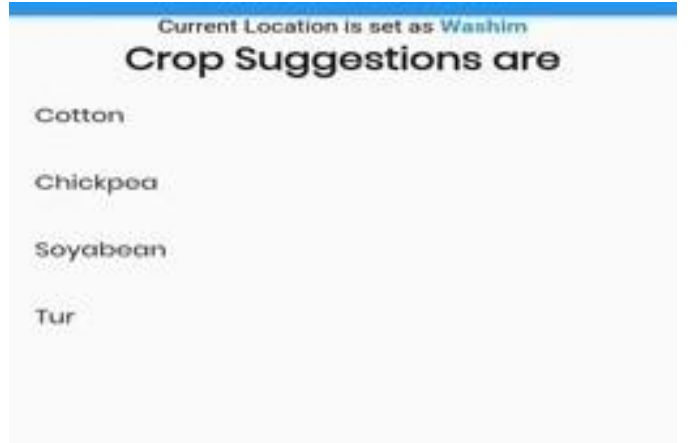

Fig 8: Crop prediction based on location and soil characteristics

The proposed system also records the current temperature upon which it suggests how much water has to be irrigated to a farm. It suggests amount of water in litre based on the size of farm in square meter and the current temperature value.

The result of water irrigation system is shown in Fig. 9.

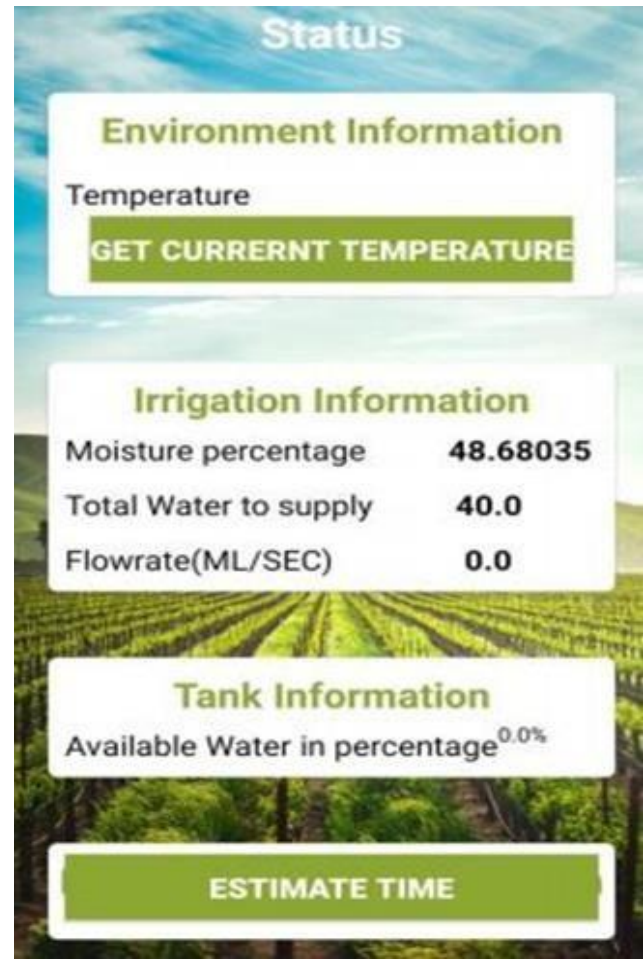

Fig 9: Result of water irrigation system

The proposed solution also gives suggestions about the appropriate organic fertilizer required to grow a crop and to keep it away from the insects and plant diseases. The selection of crop for getting fertilizer detail is shown in Fig. 10. 


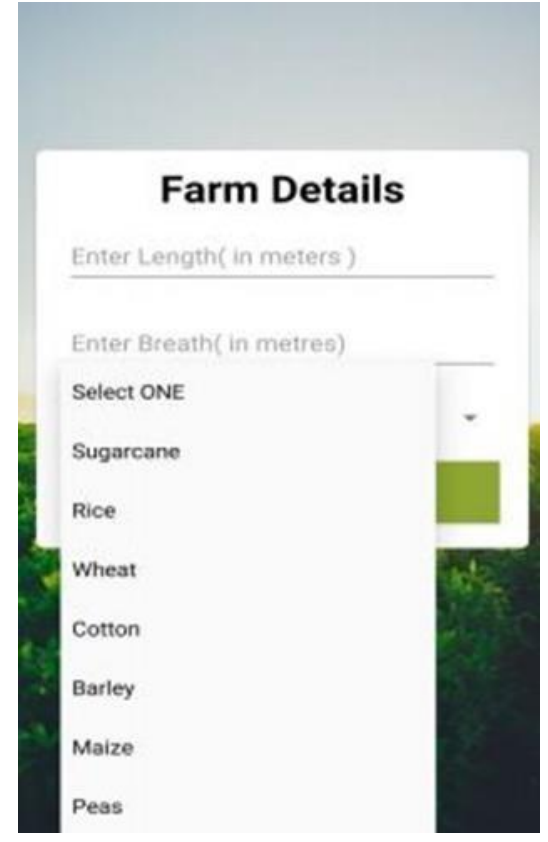

Fig 10: Selection of crop for getting fertilizer detail

The amount of organic fertilizer required to grow a selected crop is shown in Fig. 11.

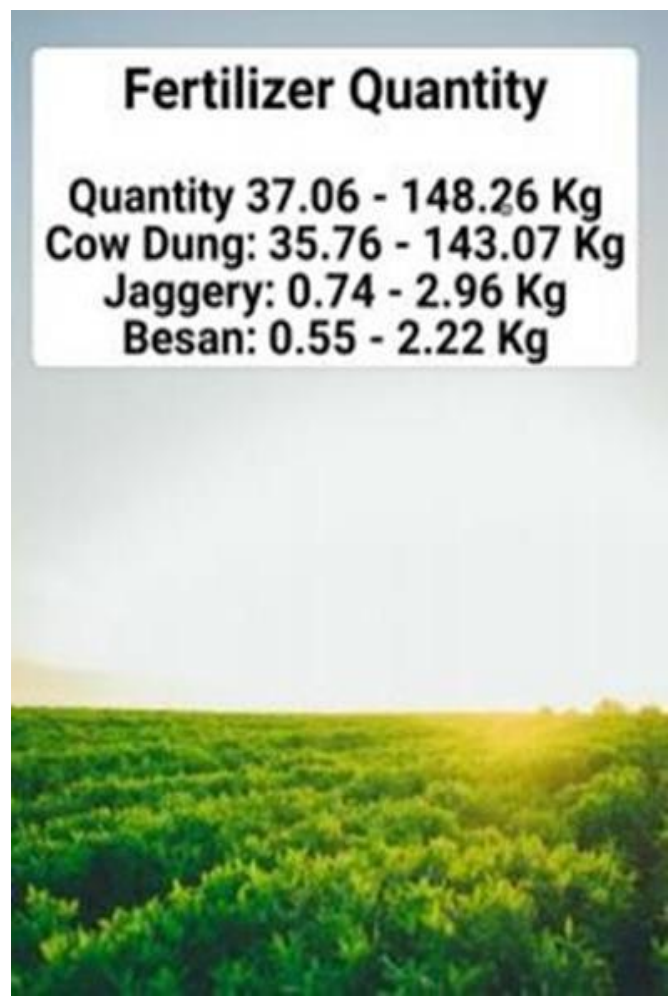

Fig 11: Amount of organic fertilizer required to grow a selected crop

In this way, the results of proposed solution implies that the farmer can have many new benefits which were never before. The results of proposed system can be used to make farming process easier, efficient and automated along with addressing all the limitations of traditional farming process.
The Smart farming system applies to agriculture, greenhouses, vineyards and plantations. It is also suitable for use in parks and in small garden houses. It can also pass on data to the user in real time. For commercial plants such as sugarcane, tobacco etc. the device may be used. It can be used for indoor and potted gardens as well. It will contribute to water management by providing the necessary quantity of water for crops, which prevents overwatering. The irrigation system, which decreases manual labor dependence and improves productivity, is automated.

\section{CONCLUSION}

The problem of traditional agriculture system, especially in India, depends on manual farming. This definition tackles unplanned schemes for water storage and strengthens existing irrigation practices. A farming system based on IoT and ML often keeps farmers alert to the coming weather and gives them the easiest advice on irrigation methods and crops, which leads to better yields. In order to diagnose basic plant conditions and spray insecticides or pesticides, the proposed model can be further enhanced. The proposed solution also has low-cost designs that take into account Indian income per capita and wealth disparities. Finally, a Smart farming design with recommendations from ML would provide farmer with a substantial education and help reduce costs by replacing Smart and downstream information with far more accurate ML-driven models. It makes it so that hundreds of millions of people have to be able to solve a very big and reliable problem. Implementing such a system on the ground can certainly help to increase crop yield and overall performance.

\section{REFERENCES}

[1] Fahad Kamraan Syed, Agniswar Paul, Ajay Kumar, Jaideep Cherukuri. "Low-cost IoT+ML design for smart farming with multiple applications", 2019 10th International Conference on Computing, Communication and Networking Technologies (ICCCNT), 2019

[2] S. Geetha, P. Deepalakshmi, Shilpa Pande."Managing Crop for Indian Farming Using IOT", 2019 IEEE International Conference on Clean Energy and Energy Efficient Electronics Circuit for Sustainable Development (INCCES), 2019

[3] Kasara Sai Pratyush Reddy, Y Mohana Roopa, Kovvada Rajeev L N, Narra Sai Nandan. "IoT based Smart Agriculture using Machine Learning", 2020 Second International Conference on Inventive Research in Computing Applications (ICIRCA), 2020

[4] Rao R., and Sridhar B. (2018). IoT based smart cropfield monitoring and automation irrigation system. $2 \mathrm{Nd}$ International Conference on Inventive Systems and Control (ICISC). doi: 10.1109/icisc.2018.8399118.

[5] Mishra D., Khan A., Tiwari R., and Upadhay S. (2018). "Automated Irrigation System-IoT Based Approach". 3rd International Conference on Internet of Things: Smart Innovation and Usages (IoT-SIU)

[6] Aashu Bedrae R. K1, Jayalakshmi A2, Nayana M3, Swetha D4, Shridhara Y5. "Development of Smart Irrigation System", June 2018 International Research Journal of Engineering and Technology (IRJET).

[7] "Future of Agriculture", https://www.iotsworld congress. com/

[8] https://agritech.tnau.ac.in 
[9] Hira Farooq, Hafeez UR Rehman et al.,", A Review on Smart IoT Based Farming", Annals of Emerging Technologies in Computing (AETiC) Vol. 4, No. 3, 2020.
[10] M.S.D. Abhiram, Jyothsnavi Kuppili, N.Alivelu Manga. "Smart Farming System using IoT for Efficient Crop Growth", 2020 IEEE International Students' Conference on Electrical, Electronics and Computer Science (SCEECS), 2020. 